\title{
Fast magnetohydrodynamic oscillation of longitudinally inhomogeneous prominence threads: an analogue with quantum harmonic oscillator
}

\author{
S. N. Lomineishvilii ${ }^{1,4}$, T. V. Zaqarashvili ${ }^{1,4}$, I. Zhelyazkov ${ }^{2}$, and A. G. Tevzadze ${ }^{3}$ \\ ${ }^{1}$ Space Research Institute, Austrian Academy of Sciences, 8042 Graz, Austria \\ e-mail: teimuraz.zaqarashvili@oeaw.ac .at \\ 2 Faculty of Physics, Sofia University, 5 James Bourchier Blvd., 1164 Sofia, Bulgaria \\ ${ }^{3}$ Faculty of Exact and Natural Sciences, Javakhishvili Tbilisi State University, 1 Chavchavadze avenue, 0128 Tbilisi, Georgia \\ ${ }^{4}$ Abastumani Astrophysical Observatory at Ilia State University, 3/5 Cholokashvili avenue, 0162 Tbilisi, Georgia
}

Received 10 December 2013 / Accepted 18 March 2014

\begin{abstract}
Context. Previous works indicate that the frequency ratio of second and first harmonics of kink oscillations tends towards 3 in the case of prominence threads. This is not a straightforward result, so it requires adequate explanation.

Aims. We aim to study the magnetohydrodynamic oscillations of longitudinally inhomogeneous prominence threads and to shed light on the problem of the frequency ratio.

Methods. The classical Sturm-Liouville problem is used for the threads with longitudinally inhomogeneous plasma density. We show that the spatial variation of total pressure perturbations along the thread is governed by the stationary Schrödinger equation, where the longitudinal inhomogeneity of plasma density stands for the potential energy. The Schrödinger equation appears as the equation of quantum harmonic oscillator for a parabolic profile of plasma density. Consequently, the equation has bounded solutions in terms of Hermite polynomials.

Results. Boundary conditions at the thread surface lead to a transcendental dispersion equation with Bessel functions. The thin flux tube approximation of the dispersion equation shows that the frequency of kink waves is proportional to the expression $\alpha(2 n+1)$, where $\alpha$ is the density inhomogeneity parameter, and $n$ the longitudinal mode number. Consequently, the ratio of the frequencies of second and first harmonics tends to 3 in prominence threads. The numerical solution of the dispersion equation shows that the ratio decreases only slightly for thicker tubes in the case of less longitudinal inhomogeneity of the external density, therefore the thin flux tube limit is a good approximation for prominence oscillations. However, stronger longitudinal inhomogeneity of external density may lead to the significant shift in the frequency ratio for wider tubes, and therefore the thin tube approximation may fail.

Conclusions. The tendency of frequency ratio of second and first harmonics towards 3 in prominence threads is explained by the analogy of the oscillations with quantum harmonic oscillator, where the density inhomogeneity of the threads plays a role as the potential energy.
\end{abstract}

Key words. Sun: filaments, prominences - Sun: oscillations - magnetohydrodynamics (MHD)

\section{Introduction}

Filaments or prominences are cold clouds of dense plasma imbedded in the tenuous hot solar corona, probably supported by the prominence magnetic field against the gravity. Observations show that prominences are made up of many tube-like fine structures that are very likely smaller than the currently achievable resolution $(\approx 150 \mathrm{~km})$. The structures are called fibrils or threads, and they have a length of $10^{3}-10^{4} \mathrm{~km}$, which is much shorter than the longitudinal extent of the prominence itself (Lin et al. 2005, Labrosse et al. 2010). Therefore, it is likely that the threads are concentrations of cold plasma at the middle of a much longer magnetic tube, while the remaining part of the tube is filled with hot coronal plasma.

Small-amplitude magnetohydrodynamic (MHD) waves and oscillations are frequently observed in solar prominence threads (Oliver et al. 2002; Lin et al. 2005, 2007, 2009; Mackay et al. 2010; Arregui et al. 2012). MHD oscillations in prominences are well studied by both slab and tube approximations
(Roberts 1991; Joarder \& Roberts 1992a, 1992b; Oliver et al. 1993; Joarder et al. 1997; Díaz et al. 2002, 2005; Dymova \& Ruderman 2005; Terradas et al. 2008; Oliver 2009; Arregui et al. 2011, 2012). It turns out that the longitudinal density stratification significantly influences the oscillation spectra of simple homogeneous tubes (Díaz et al. 2004, 2010; Andries et al. 2005a, 2009; Dymova \& Ruderman 2005; McEwan et al. 2006; Zaqarashvili et al. 2007, 2013).

However, the oscillations in coronal loops and prominence threads are very different. The difference is caused by the density profile: coronal loops have denser plasma at footpoints probably owing to the stratification, while prominence threads (fibrils) are denser at the midpoint of magnetic tube (or slab). The density contrast between the tube centre and footpoints is by an order larger in prominences than in coronal loops. The different physical parameters lead to different oscillation spectra in these two structures. For example, the ratio between the periods of first and second harmonics in homogeneous coronal loops is near 2 (Edwin \& Roberts 1983), while the stratification may lead to the 
significant shift in the ratio (Andries et al. 2005b; McEwan et al. 2006). There are several observational examples of the simultaneous existence of first and second harmonics of MHD oscillations in coronal loops, which indeed show the deviation of the ratio from 2 (Van Doorsselaere et al. 2004, 2007; Verwichte et al. 2004; De Moortel \& Brady 2007; Srivastava et al. 2008, 2013; Inglis \& Nakariakov 2009).

On the other hand, the period ratio of first and second harmonics is not 2 in the prominence case. Early calculations showed that the asymptotic frequency of internal kink mode in the prominence case is proportional to $2 n+1$, where $n$ is the mode number (Joarder \& Roberts 1992b). Then, the period ratio of first and second harmonics is 3 , in contrast to coronal loops. The similar ratio can be seen on the plots of other papers concerning the prominence oscillations (Dymova \& Ruderman 2005; Díaz et al. 2010). However, the authors did not clearly note this and consequently did not explain why the period ratio tends towards 3 in prominences. The frequency dependence of $2 n+1$ found by Joarder \& Roberts (1992b) suggests that the quantum mechanical analogy may support the result. All these authors used piecewise profiles to study the prominence oscillations. Consequently, the concentration of density in the middle of tube can be considered as potential energy, which may lead to the problem of quantum harmonic oscillator.

In this paper, we reconsider the oscillation of prominence threads using a parabolic density profile in the longitudinal direction. The solution of Sturm-Liouville problem allows us to obtain the equation of a quantum harmonic oscillator. The solution of the equation leads to the frequency dependence of $2 n+1$, so we may conclude that the enhancement of plasma density (both piecewise and parabolic profiles) at the middle of flux tube allows waves to be quantified analogously with quantum mechanics.

The paper is organized as follows. In Sect. 2 we describe the physical model of the considered problem. In Sect. 3 we give the Sturm-Liouville solution that leads to the dispersion equation described in Sect. 4. Analytical and numerical solutions of the dispersion equation are given in Sects. 4.1 and 4.2. We briefly summarize the paper in Sect. 5 .

\section{Main equations}

We consider a prominence thread as a magnetic tube of length $2 L$ and radius $a$ in the cylindrical coordinate system $(r, \varphi, z)$. We assume that the unperturbed density inside the tube is homogenous along the radial direction and has longitudinal parabolic inhomogeneity along the $z$-axis in the form of

$\rho_{0}(r<a)=\rho_{\mathrm{i}}=\rho_{\mathrm{i} 0}\left(1-\alpha^{2} \frac{z^{2}}{L^{2}}\right)$,

where $\alpha$ is a constant, and $\rho_{\mathrm{i} 0}$ the unperturbed density at the tube midpoint (i.e. at $z=0$ ) inside the thread, which has a typical value for prominences. The thread is imbedded in a coronal plasma with much lower density. The density is higher at the tube midpoint and decreases towards the ends approaching its coronal value (Fig. 1). The thread itself probably is a part of much longer magnetic tube, which has a coronal density outside the thread. The magnetic field is uniform and longitudinal; i.e., the tube is untwisted.

We consider a cold plasma approximation without gravity, then the single-fluid, linear ideal MHD equations can be written in cylindrical coordinates as

$$
\begin{aligned}
& \frac{\partial u_{r}}{\partial t}=\frac{B_{0}}{4 \pi \rho_{0}} \frac{\partial b_{r}}{\partial z}-\frac{B_{0}}{4 \pi \rho_{0}} \frac{\partial b_{z}}{\partial r}, \\
& \frac{\partial u_{\varphi}}{\partial t}=\frac{B_{0}}{4 \pi \rho_{0}} \frac{\partial b_{\varphi}}{\partial z}-\frac{B_{0}}{4 \pi \rho_{0} r} \frac{\partial b_{z}}{\partial \varphi}, \\
& \frac{\partial b_{r}}{\partial t}=B_{0} \frac{\partial u_{r}}{\partial z}, \\
& \frac{\partial b_{\varphi}}{\partial t}=B_{0} \frac{\partial u_{\varphi}}{\partial z}, \\
& \frac{\partial b_{z}}{\partial t}=-B_{0} \frac{u_{r}}{r}-B_{0} \frac{\partial u_{r}}{\partial r}-\frac{B_{0}}{r} \frac{\partial u_{\varphi}}{\partial \varphi},
\end{aligned}
$$

where $\rho_{0}$ is the unperturbed density, $B_{0}$ the unperturbed magnetic field, and $b_{r}, b_{\varphi}, b_{z}, u_{r}, u_{\varphi}$ are magnetic field and velocity perturbations, respectively. The longitudinal velocity component is zero; i.e., $u_{z}=0$, in $\beta\left(=4 \pi p / B^{2}\right)=0$ approximation. Equations (2)-(6) represent a closed set of equations, therefore the continuity equation is not necessary here. Density perturbations can be expressed by magnetic field perturbations later, if necessary.

Equations (2)-(6) lead, after some algebra, to the following equation

$\frac{1}{r} \frac{\partial}{\partial r}\left(r \frac{\partial p_{\mathrm{m}}}{\partial r}\right)+\frac{1}{r^{2}} \frac{\partial^{2} p_{\mathrm{m}}}{\partial \varphi^{2}}+\frac{\partial^{2} p_{\mathrm{m}}}{\partial z^{2}}-\frac{1}{v_{\mathrm{A}}^{2}} \frac{\partial^{2} p_{\mathrm{m}}}{\partial t^{2}}=0$,

where

$p_{\mathrm{m}}=\frac{B_{0} b_{z}}{4 \pi}$

is the perturbed magnetic pressure, and

$v_{\mathrm{A}}=\frac{B_{0}}{\sqrt{4 \pi \rho_{0}}}$

is the Alfvén speed.

To find the spectrum of MHD oscillations in a magnetic tube, one should solve Eq. (7) inside and outside the tube and then merge the solutions at the tube surface through the continuity of the total pressure and radial velocity.

\section{Sturm-Liouville problem and Schrödinger equation}

Fourier analysis of Eq. (7) with $\exp (-\mathrm{i} \omega t+\mathrm{i} m \varphi)$ using Eq. (1) leads to the following equation inside the tube

$\frac{1}{r} \frac{\partial}{\partial r}\left(r \frac{\partial p_{\mathrm{m}}}{\partial r}\right)+\frac{\partial^{2} p_{\mathrm{m}}}{\partial z^{2}}+\left[\frac{\omega^{2}}{v_{\mathrm{Ai} 0}^{2}}\left(1-\frac{\alpha^{2} z^{2}}{L^{2}}\right)-\frac{m^{2}}{r^{2}}\right] p_{\mathrm{m}}=0$

where

$v_{\mathrm{Ai} 0}=\frac{B_{0}}{\sqrt{4 \pi \rho_{\mathrm{i} 0}}}$

is the Alfvén speed at $z=0$.

We assume that the density outside the tube has the parabolic profile along $z$-axis as inside the tube, namely

$\rho_{0}(r>a)=\rho_{\mathrm{e}}=\rho_{\mathrm{e} 0}\left(1-\alpha_{\mathrm{e}}^{2} \frac{z^{2}}{L^{2}}\right)$, 


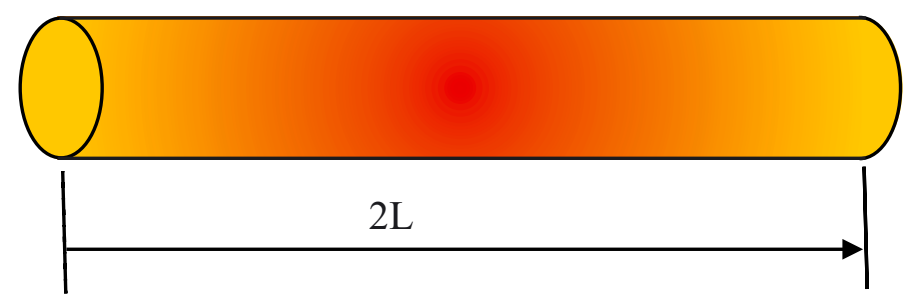

Fig. 1. Prominence thread with a longitudinal inhomogeneity of plasma density.

where $\alpha_{\mathrm{e}}$ is a constant and $\rho_{\mathrm{e} 0}$ is unperturbed density at $z=0$ outside the tube. Then the equation similar to Eq. (10) is obtained outside the tube

$\frac{1}{r} \frac{\partial}{\partial r}\left(r \frac{\partial p_{\mathrm{m}}}{\partial r}\right)+\frac{\partial^{2} p_{\mathrm{m}}}{\partial z^{2}}+\left[\frac{\omega^{2}}{v_{\mathrm{Ae} 0}^{2}}\left(1-\frac{\alpha_{\mathrm{e}}^{2} z^{2}}{L^{2}}\right)-\frac{m^{2}}{r^{2}}\right] p_{\mathrm{m}}=0$,

where

$v_{\mathrm{Ae} 0}=\frac{B_{0}}{\sqrt{4 \pi \rho_{\mathrm{e} 0}}}$.

Equations (10) and (13) with corresponding boundary conditions represent a classical Sturm-Liouville problem, and they can be solved by the method of separation of variables.

We assume

$p_{\mathrm{m}}(r, z)=p_{\mathrm{m} r}(r) p_{\mathrm{m} z}(z)$.

Then Eq. (10) leads to the following equations

$$
\begin{aligned}
& \frac{1}{r} \frac{\partial}{\partial r}\left(r \frac{\partial p_{\mathrm{m} r}}{\partial r}\right)+\left(\frac{\omega^{2}}{v_{\mathrm{Ai} 0}^{2}}-\frac{m^{2}}{r^{2}}\right) p_{\mathrm{m} r}=k_{n}^{2} p_{\mathrm{m} r}, \\
& \frac{\partial^{2} p_{\mathrm{m} z}}{\partial z^{2}}-\frac{\omega^{2}}{v_{\mathrm{Ai} 0}^{2}} \alpha^{2} \frac{z^{2}}{L^{2}} p_{\mathrm{m} z}=-k_{n}^{2} p_{\mathrm{m} z}
\end{aligned}
$$

where $k_{n}^{2}$ is the separation constant.

Equation (17) is analogous to the stationary Schrödinger equation, where the first and second terms in the left-hand side correspond to the kinetic and potential energies (after corresponding normalization), while the term in the right hand side corresponds to the total energy. More specifically, with substitution of

$\alpha /\left(v_{\mathrm{Ai} 0} L\right)=m / \hbar, k_{n}=\sqrt{2 m E} / \hbar, p_{\mathrm{m} z}=\Psi(z)$,

Eq. (17) is rewritten as

$\frac{-\hbar^{2}}{2 m} \frac{\partial^{2} \Psi(z)}{\partial z^{2}}+\frac{1}{2} m \omega^{2} z^{2} \Psi(z)=E \Psi(z)$

which is the equation of a quantum harmonic oscillator (Griffiths 2004). It must be noted that the considered problem is entirely classical: the Planck's constant is introduced here just to demonstrate the mathematical similarity of the considered problem with the behaviour of a quantum particle in a potential well. This equation has exact solutions and governs many dynamical systems in different branches of science.

Assuming $\alpha=0$ in Eq. (17) leads to the solution of homogeneous tube, and after corresponding calculation, one may recover well known dispersion relations obtained by Edwin \& Roberts (1983).

Now we introduce a new variable

$x \equiv z \sqrt{\frac{2 \alpha \omega}{v_{\mathrm{Ai} 0} L}}$ then, from Eq. (17) we obtain the equation of parabolic cylinder

$\frac{\partial^{2} p_{\mathrm{m} z}}{\partial x^{2}}-\left(\frac{x^{2}}{4}+d\right) p_{\mathrm{m} z}=0$

where

$d \equiv-\frac{v_{\mathrm{Ai} 0} L}{2 \alpha \omega} k_{n}^{2}<0$,

for $\omega>0$. For $\omega<0$, we have $d>0$.

After the substitution of Eq. (20), it is no longer possible to recover the dispersion relation of homogeneous tube. The approximation of homogeneous tube means $\alpha=0$, which implies that $x=0$ everywhere, so Eq. (21) is not determined. The solutions of Eq. (21) are parabolic cylinder or Weber functions $U(d, x)$ and $V(d, x)$ (Abramowitz \& Stegun 1964).

When

$d=-n-\frac{1}{2}$,

where $n=0,1,2, \ldots$, the bounded solutions can be expressed in terms of Hermite polynomials (Abramowitz \& Stegun 1964):

$U\left(-n-\frac{1}{2}, x\right)=2^{-\frac{n}{2}} \mathrm{e}^{-\frac{x^{2}}{4}} H_{n}\left(\frac{x}{\sqrt{2}}\right)$,

for $\omega>0$ and

$V\left(n+\frac{1}{2}, x\right)=2^{-\frac{n}{2}} \mathrm{e}^{\frac{x^{2}}{4}}(-\mathrm{i})^{n} H_{n}\left(\frac{\mathrm{i} x}{\sqrt{2}}\right)$,

for $\omega<0$. The Hermite polynomials are a set of orthogonal polynomials over the domain $(-\infty, \infty)$ with weighting function $\exp \left(x^{2} / 2\right)$, therefore the functions $U\left(-n-\frac{1}{2}, x\right)$ and $V\left(n+\frac{1}{2}, x\right)$ form an orthogonal basis of the Hilbert space.

Equations (22) and (23) imply that

$k_{n}^{2}=\frac{\alpha \omega}{v_{\mathrm{Ai} 0} L}(2 n+1)$.

Equations (18) and (26) lead to the equation

$E_{n}=\hbar \omega_{n}\left(n+\frac{1}{2}\right)$

which is a well known formula for energy levels, and thus the oscillation of prominence threads is analogous with quantum harmonic oscillator. Nakariakov \& Oraevsky (1995) also studied a plasma non-uniformity with a parabolic (transverse) profile in the context of MHD oscillations of coronal plasma structures and the solutions of a boundary problem in terms of the Hermite polynomials with the use of the quantum mechanical analogy were found.

From Eq. (26) it is seen that $\omega<0$ leads to imaginary $k_{n}$, therefore the solution can be neglected for physical reasons. Accordingly, in the future we only consider the solution with $\omega>0$, i.e., Eq. (24). This solution rapidly decreases along $x$ due to the factor of $\mathrm{e}^{-x^{2} / 4}$, hence it is bounded in the longitudinal direction. As a result, there is no need to fix close boundary conditions at the end of a much longer tube as usually done for piecewise profiles (Dymova \& Ruderman 2005; Díaz et al. 2010). Density inhomogeneity of prominence thread traps these bounded oscillations analogously to quantum harmonic oscillator. 
The general solution of Eq. (21) is

$p_{\mathrm{m} z}=p_{\mathrm{m} z}(0) 2^{-\frac{n}{2}} \mathrm{e}^{-\frac{x^{2}}{4}} H_{n}\left(\frac{x}{\sqrt{2}}\right)$

where $p_{\mathrm{m} z}(0)$ is a constant. Now, Eq. (16) can be rewritten as

$\frac{1}{r} \frac{\partial}{\partial r}\left(r \frac{\partial p_{\mathrm{m} r \mathrm{i}}}{\partial r}\right)+\left[m_{\mathrm{i}}^{2}-\frac{m^{2}}{r^{2}}\right] p_{\mathrm{m} r \mathrm{i}}=0$,

where $p_{\text {mri }}$ is the radially dependent part of the total pressure perturbations inside the tube and

$m_{\mathrm{i}}^{2}=\frac{\omega^{2}}{v_{\mathrm{Ai} 0}^{2}}-\frac{\alpha \omega}{v_{\mathrm{Ai} 0} L}(2 n+1)$.

Repeating the same calculation outside the tube, we obtain the equation

$\frac{1}{r} \frac{\partial}{\partial r}\left(r \frac{\partial p_{\mathrm{m} r \mathrm{e}}}{\partial r}\right)-\left[m_{\mathrm{e}}^{2}+\frac{m^{2}}{r^{2}}\right] p_{\mathrm{m} r \mathrm{e}}=0$,

where $p_{\text {mre }}$ is the radially dependent part of the total pressure perturbations outside the tube, and

$m_{\mathrm{e}}^{2}=\frac{\alpha_{\mathrm{e}} \omega}{v_{\mathrm{Ae} 0} L}(2 n+1)-\frac{\omega^{2}}{v_{\mathrm{Ae} 0}^{2}}$.

Equations (29) and (31) are Bessel and modified Bessel equations, respectively. Therefore, the corresponding solutions are

$p_{\mathrm{m} r \mathrm{i}}=a_{1} J_{m}\left(m_{\mathrm{i}} r\right)+a_{2} Y_{m}\left(m_{\mathrm{i}} r\right)$

$p_{\text {mre }}=b_{1} I_{m}\left(m_{\mathrm{e}} r\right)+b_{2} K_{m}\left(m_{\mathrm{e}} r\right)$,

where $a_{1}, a_{2}, b_{1}, b_{2}$ are constants.

Total pressure perturbation inside the tube, $p_{\text {mri }}$, must be finite at $r=0$, which yields $a_{2}=0$. Analogously, total pressure perturbation outside the tube $p_{\text {mre }}$ must be finite at $r \rightarrow \infty$, which yields $b_{1}=0$. These two conditions lead to the following expressions of total pressure perturbations inside and outside the tube:

$p_{\mathrm{m} r \mathrm{i}}=a_{1} J_{m}\left(m_{\mathrm{i}} r\right)$,

$p_{\mathrm{m} r \mathrm{e}}=b_{2} K_{m}\left(m_{\mathrm{e}} r\right)$.

\section{Dispersion equation}

The dispersion equation for MHD oscillations in the magnetic tube can be obtained through the boundary conditions at the tube surface. The boundary conditions require the continuity of total pressure and radial velocity at $r=a$. Total pressure balance condition at the tube surface yields

$\left[p_{\mathrm{m} r \mathrm{i}}\right]_{a}=\left[p_{\mathrm{mre}}\right]_{a} \Rightarrow a_{1} J_{m}\left(m_{\mathrm{i}} a\right)=b_{2} K_{m}\left(m_{\mathrm{e}} a\right)$.

The equations for radial velocity perturbations inside the tube can be obtained from Eq. (2) as

$\frac{\partial^{2} u_{r \mathrm{i}}}{\partial z^{2}}+\frac{\omega^{2}}{v_{\mathrm{Ai}}^{2}} u_{r \mathrm{i}}=-\frac{\mathrm{i} \omega}{\rho_{\mathrm{i}} v_{\mathrm{Ai}}^{2}} \frac{\partial p_{\mathrm{mi}}}{\partial r}$.

Next, we assume

$u_{r \mathrm{i}}(r, z)=u_{r \mathrm{i}}(r) p_{\mathrm{mz}}(z)$,

then, Eq. (38) leads to the following equation:

$u_{r \mathrm{i}}(r)\left[\frac{\partial^{2} p_{\mathrm{m} z}(z)}{\partial z^{2}}+\frac{\omega^{2}}{v_{\mathrm{Ai}}^{2}} p_{\mathrm{m} z}(z)\right]=-\frac{\mathrm{i} \omega p_{\mathrm{m} z}(z)}{\rho_{\mathrm{i}} v_{\mathrm{Ai}}^{2}} \frac{\partial p_{\mathrm{m} r \mathrm{i}}(r)}{\partial r}$.
Using Eq. (17) this equation can be rewritten after some algebra as

$u_{r \mathrm{i}}(r)=-\frac{\mathrm{i} \omega}{\rho_{\mathrm{i} 0}\left(\omega^{2}-v_{\mathrm{Ai} 0}^{2} k_{n}^{2}\right)} \frac{\partial p_{\mathrm{m} r \mathrm{i}}(r)}{\partial r}$.

Similar calculations outside the tube leads to the equation

$u_{r \mathrm{e}}(r)=-\frac{\mathrm{i} \omega}{\rho_{\mathrm{e} 0}\left(\omega^{2}-v_{\mathrm{Ae} 0}^{2} k_{n \mathrm{e}}^{2}\right)} \frac{\partial p_{\mathrm{m} r \mathrm{e}}(r)}{\partial r}$,

where

$k_{n \mathrm{e}}^{2}=\frac{\alpha_{\mathrm{e}} \omega}{v_{\mathrm{Ae} 0} L}(2 n+1)$.

The continuity of transverse velocity at the tube surface yields

$\rho_{\mathrm{e} 0}\left(\omega^{2}-v_{\mathrm{Ae} 0}^{2} k_{n \mathrm{e}}^{2}\right)\left[\frac{\partial p_{\mathrm{m} r \mathrm{i}}}{\partial r}\right]_{a}=\rho_{\mathrm{i} 0}\left(\omega^{2}-v_{\mathrm{Ai} 0}^{2} k_{n}^{2}\right)\left[\frac{\partial p_{\mathrm{m} r \mathrm{e}}}{\partial r}\right]_{a}$

With the help of Eqs. (35)-(37) we obtain the final transcendental dispersion equation

$R_{\mathrm{e} 0} \frac{J_{m}^{\prime}\left(R_{\mathrm{i} 0}\right)}{J_{m}\left(R_{\mathrm{i} 0}\right)}=-R_{\mathrm{i} 0} \frac{K_{m}^{\prime}\left(R_{\mathrm{e} 0}\right)}{K_{m}\left(R_{\mathrm{e} 0}\right)}$,

where the prime sign, ', indicates a differentiation by Bessel function argument and

$R_{\mathrm{i} 0}=\xi \sqrt{\tilde{\omega}^{2}-\alpha \tilde{\omega}(2 n+1)}$,

$R_{\mathrm{e} 0}=\xi \sqrt{\alpha_{\mathrm{e}} \tilde{\omega} \sqrt{\eta}(2 n+1)-\eta \tilde{\omega}^{2}}$,

where

$\xi=\frac{a}{L}, \quad \tilde{\omega}=\frac{\omega L}{v_{\mathrm{Ai} 0}}, \quad \eta=\frac{\rho_{\mathrm{e} 0}}{\rho_{\mathrm{i} 0}}$.

Here $m=0$ and $m=1$ correspond to the sausage and kink waves, respectively, while $n=0(n=1)$ is the first (second) harmonic.

\subsection{Analytical solution}

The thin flux tube approximation, i.e., $\xi=a / L \ll 1$ yields $R_{\mathrm{i} 0} \ll 1$ and $R_{\mathrm{e} 0} \ll 1$. Then, using the recurrence relations of the Bessel functions, $J_{m}^{\prime}(z)=-J_{m+1}(z)+(m / z) J_{m}(z)$ and $K_{m}^{\prime}(z)=-K_{m+1}(z)+(m / z) K_{m}(z)$, and the expansion of Bessel functions for small arguments (Abramowitz \& Stegun 1964)

$J_{m}(z) \sim\left(\frac{z}{2}\right)^{m} \frac{1}{\Gamma(m+1)}, m \neq-1,-2,-3, \ldots$,

$K_{m}(z) \sim \frac{1}{2} \Gamma(m)\left(\frac{z}{2}\right)^{-m}, \operatorname{Re}(m)>0$.

Equation (45) can be approximated for kink waves $(m=1)$ as

$\eta \xi^{2} \tilde{\omega}_{n}^{3}-\xi^{2}(2 n+1)\left(\alpha_{\mathrm{e}} \sqrt{\eta}+\alpha \eta\right) \tilde{\omega}_{n}^{2}+\left[\xi^{2} \alpha \alpha_{\mathrm{e}} \sqrt{\eta}(2 n+1)^{2}\right.$
$-4 \eta-4] \tilde{\omega}_{n}+4(2 n+1)\left(\alpha+\alpha_{\mathrm{e}} \sqrt{\eta}\right)=0$.

From this equation we obtain the following expression for the fundamental kink wave (using $\xi \ll 1$ ):

$\omega_{n} \approx \frac{v_{\mathrm{Ai} 0}}{L}(2 n+1) \frac{\alpha+\alpha_{\mathrm{e}} \sqrt{\eta}}{1+\eta}$.

Equation (52) shows that the factor $2 n+1$ appears in the expression of kink wave frequency. As we expected this is because the 

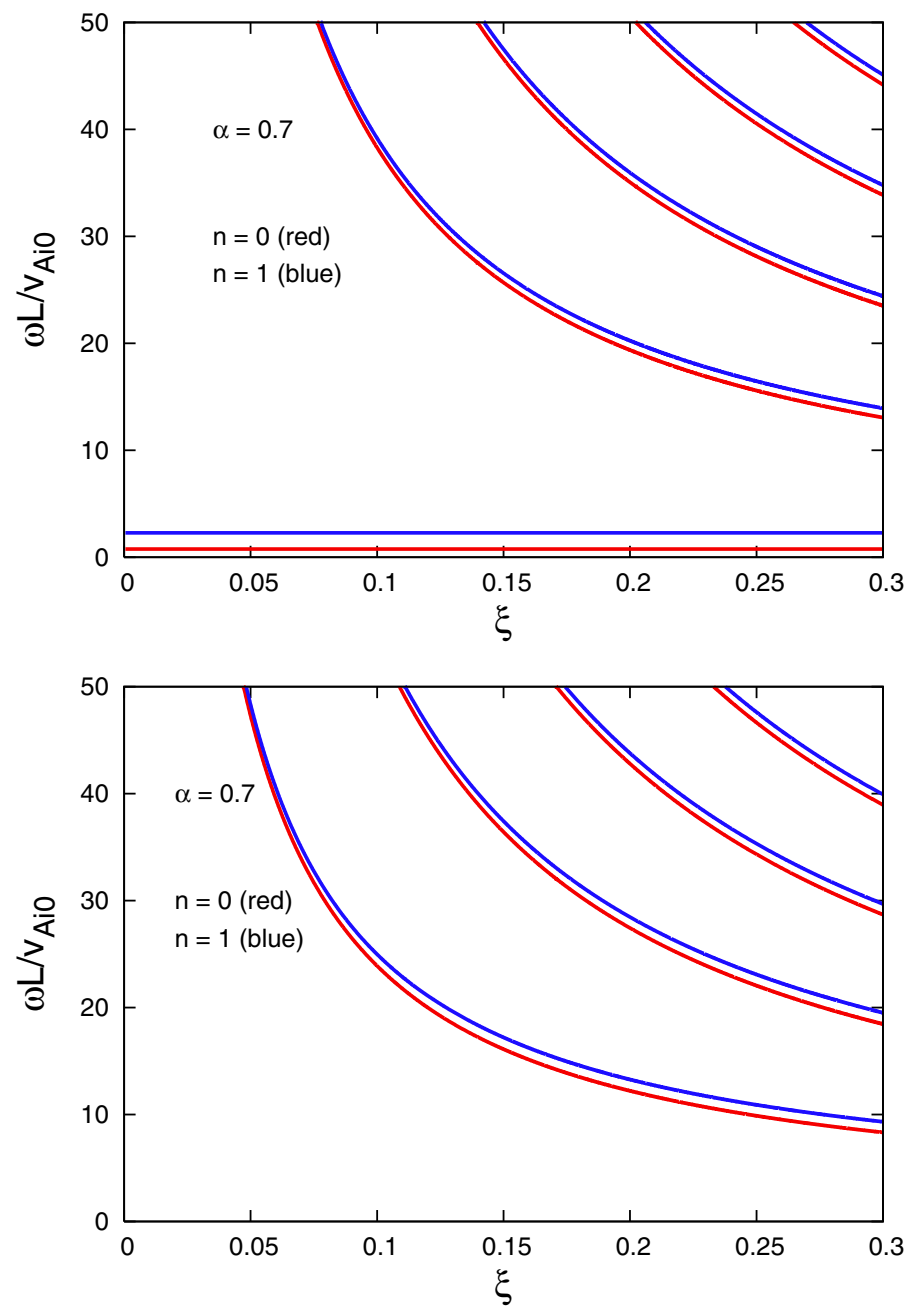

Fig. 2. Normalized frequency, $\tilde{\omega}=\omega L / v_{\mathrm{Ai} 0}$, vs the ratio of the tube width to length, $\xi=a / L$, for kink (upper panel) and sausage (lower panel) waves. $n=0$ (red lines) and $n=1$ (blue lines) correspond to the first and second harmonics. Here $\eta=0.01$ and $\alpha=\alpha_{\mathrm{e}}=0.7$ are used.

density enhancement at the tube midpoint stands for the potential energy, therefore the oscillations are similar to the case of quantum harmonic oscillator. It is also seen that the frequency of kink waves is proportional to the inhomogeneity parameters $\alpha$ and $\alpha_{\mathrm{e}}$, which stand for the depth of the potential well. The first harmonic or basic energy level of the oscillations is then

$\omega_{0} \approx \frac{v_{\mathrm{Ai} 0}}{L} \frac{\alpha+\alpha_{\mathrm{e}} \sqrt{\eta}}{1+\eta}$

while the second harmonic or second energy level is

$\omega_{1} \approx \frac{3 v_{\mathrm{Ai0}}}{L} \frac{\alpha+\alpha_{\mathrm{e}} \sqrt{\eta}}{1+\eta}$

Then the ratio of the frequencies of second and first harmonics is

$\frac{\omega_{1}}{\omega_{0}} \approx 3$.

This is the ratio that has been obtained from time to time in the case of prominence oscillations without physical explanation (Joarder \& Roberts 1992b; Dymova \& Ruderman 2005; Díaz et al. 2010).

To check the analytical solution of the dispersion relation, we use numerical solutions of Eq. (45), which are presented in the next section.

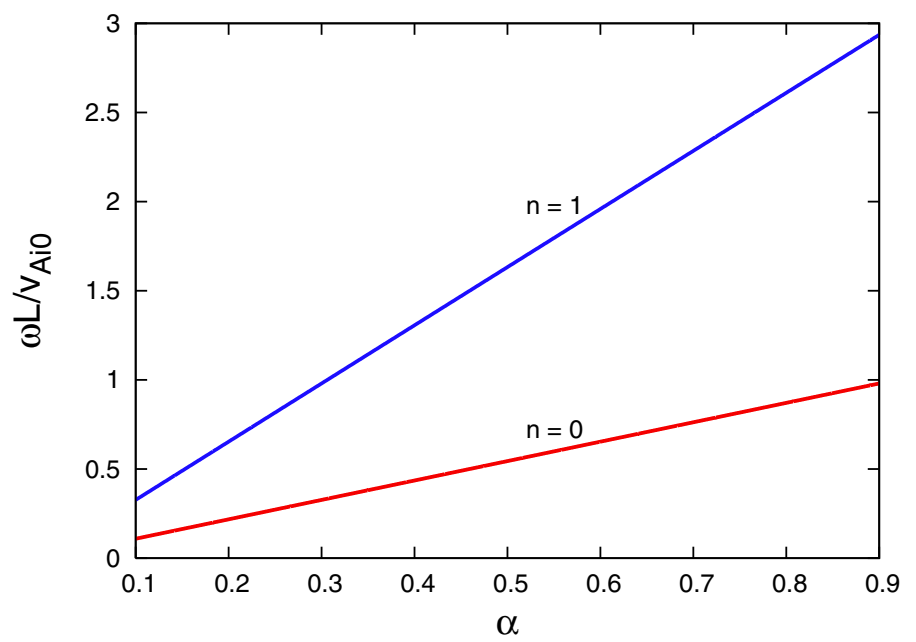

Fig. 3. Normalized frequency of first (red curve) and second (blue curve) harmonics of fundamental kink waves vs the inhomogeneity parameter, $\alpha$, for $\eta=0.01$, in the case of $\alpha_{\mathrm{e}}=\alpha$.

\subsection{Numerical solution}

We solved Eq. (45) numerically for sausage $(m=0)$ and kink $(m=1)$ modes in the prominence conditions. The inhomogeneity parameter of external density, $\alpha_{\mathrm{e}}$, may affect the wave frequencies. Therefore, we solve the dispersion Eq. (45) for two different values of $\alpha_{\mathrm{e}}$. First, we suppose that $\alpha_{\mathrm{e}}=\alpha$, so the plasma density inside and outside has same behaviour. Then we consider $\alpha_{\mathrm{e}}=\alpha / \sqrt{\eta}>\alpha$ and show how the stronger external inhomogeneity of plasma density influences the results.

Figure 2 shows the dependence of frequency of first (often called fundamental) and second harmonics on the ratio of the tube width to length, $\xi=a / L$, for $\alpha=0.7$. The plots are similar to the one of Edwin \& Roberts (1983). The fundamental kink mode is expressed by horizontal lines in the upper plot. It is seen in Fig. 2 that the frequencies of $n=0$ and $n=1$ harmonics of fundamental kink mode differs by a factor of 3 . However, the two curves are almost identical for higher harmonics which correspond to higher noughts of Bessel functions. These modes correspond to the propagation of waves almost along the radial direction, which means that their frequency is determined by the tube radius $a$, not by the tube length $L$. A similar result was found recently by Nakariakov et al. 2012 for sausage modes of a uniform cylinder. For example, the normalized frequency of second kink mode at $\xi=0.1$ is $\sim 45$, which shows that the corresponding wavelength is of the order of $a$. It means that the frequencies of $n=0$ and $n=1$ modes are determined by the tube radius $a$, therefore they have similar values.

Figure 3 shows the dependence of a fundamental kink mode frequency on the inhomogeneity parameter $\alpha$. The curves corresponding to the different values of $\xi$ are practically identical for both first and second harmonics, therefore we provide only curves for one value. On the contrary, the frequency of a fundamental kink mode depends linearly on the inhomogeneity parameter $\alpha$. This agrees with analytical formula, Eq. (52). It is also seen that the ratio of second and first harmonic frequencies is near 3 in thin and weakly inhomogeneous tubes, in complete agreement with Eq. (55).

The dependence of the frequency ratio of second and first harmonics of a fundamental kink mode on the ratio of the tube width to length is shown in Fig. 4 for different values of $\alpha$. It is seen that the frequency ratio decreases only slightly for wider tubes. The decrease is more pronounced for the tubes with 


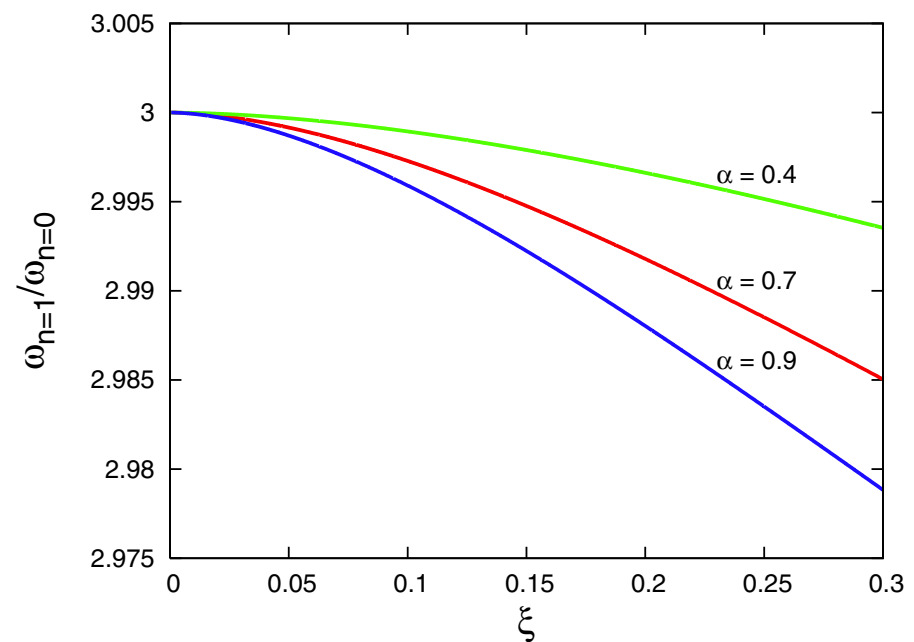

Fig. 4. Frequency ratio, $\omega_{n=1} / \omega_{n=0}$, of the kink mode vs. $\xi$ for $\eta=0.01$ and three different values of $\alpha$ equal to 0.4 (green line), 0.7 (red line), and 0.9 (blue line) in the case of $\alpha_{\mathrm{e}}=\alpha$.

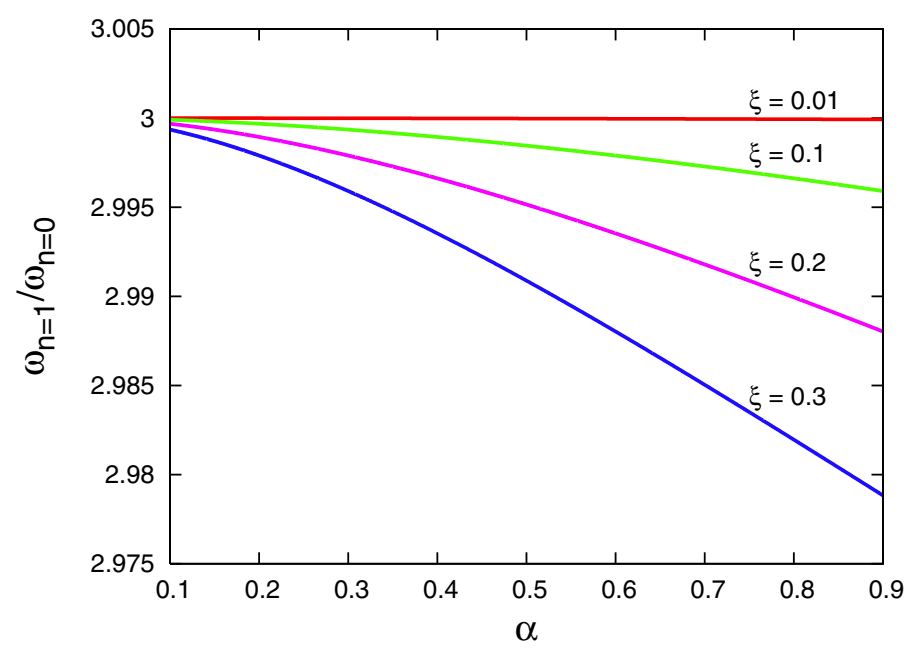

Fig. 5. Frequency ratio, $\omega_{n=1} / \omega_{n=0}$, of the kink mode vs $\alpha$ for $\eta=0.01$ and four different values of $\xi$ equal to 0.01 (red line), 0.1 (green line), 0.2 (purple line), and 0.3 (blue line) in the case of $\alpha_{\mathrm{e}}=\alpha$.

stronger longitudinal inhomogeneity, but it is still insignificant in general.

Figure 5 shows the dependence of the frequency ratio on the inhomogeneity parameter $\alpha$ for different values of $\xi$. The frequency ratio is almost constant in both, thin and thick tubes.

Now we solve the dispersion equation, Eq. (45), for the $\alpha_{\mathrm{e}}=\alpha / \sqrt{\eta}$ case. The dependence of the frequency ratio of second and first harmonics of fundamental kink mode on the ratio of tube width to length is shown in Fig. 6 for different values of $\alpha$. It is seen that the frequency ratio decreases significantly for wider tubes. The decrease is greater for the tubes with strong longitudinal inhomogeneity.

Figure 7 shows the dependence of the frequency ratio on the inhomogeneity parameter $\alpha$ for different values of $\xi$. The frequency ratio is almost constant in thin tubes, but it depends strongly on $\alpha$ for wider tubes.

Thus, the numerical simulations of the dispersion relation, Eq. (45), show that the frequency of a fundamental kink mode depends significantly on the inhomogeneity parameter, $\alpha$. On the contrary, the frequency ratio of second and first harmonics has a weak dependence on density inhomogeneity when

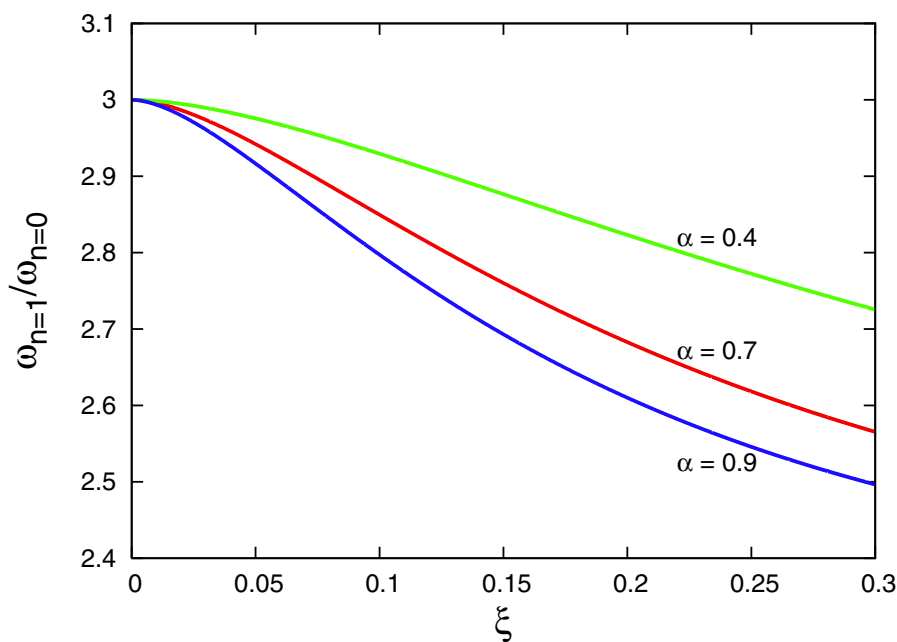

Fig. 6. Frequency ratio, $\omega_{n=1} / \omega_{n=0}$, of the kink mode vs $\xi$ for $\eta=0.01$ and three different values of $\alpha$ equal to 0.4 (green line), 0.7 (red line), and 0.9 (blue line). Here $\alpha_{\mathrm{e}}=\alpha / \sqrt{\eta}$ is assumed.

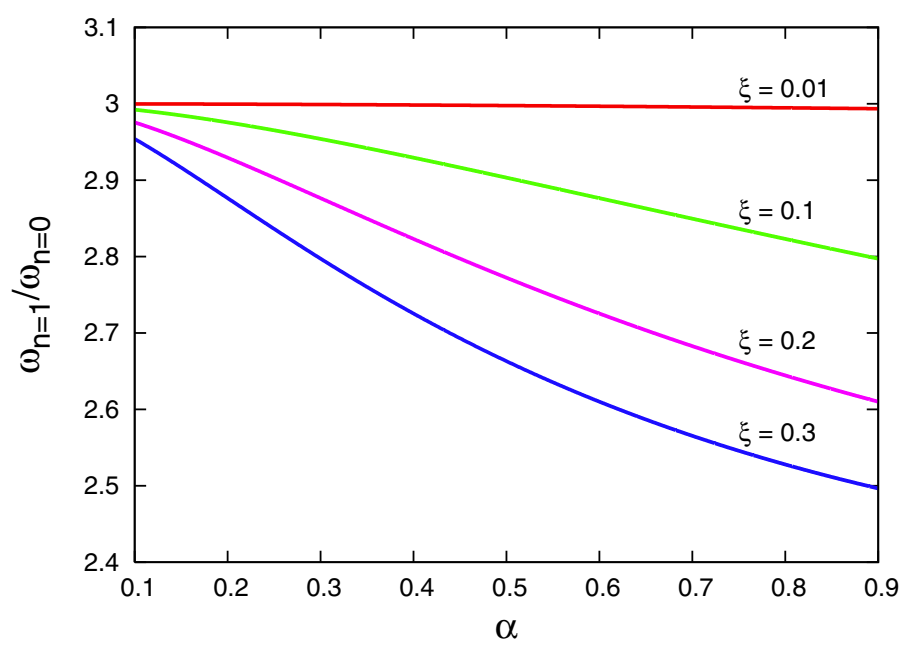

Fig. 7. Frequency ratio, $\omega_{n=1} / \omega_{n=0}$, of the kink mode vs $\alpha$ for $\eta=0.01$ and four different values of $\xi$ equal to 0.01 (red line), 0.1 (green line), 0.2 (purple line), and 0.3 (blue line). Here $\alpha_{\mathrm{e}}=\alpha / \sqrt{\eta}$ is assumed.

$\alpha_{\mathrm{e}}=\alpha$. However, when $\alpha_{\mathrm{e}}$ increases, then the frequency ratio depends notably on this parameter for wider tubes. There is a good agreement between numerical solutions and analytical formulas, Eqs. (52) and (55). Both frequency and the frequency ratio do not depend noticeably on the ratio of the tube width to length for $\alpha_{\mathrm{e}}=\alpha$. Therefore, the thin flux tube limit is a good approximation for fundamental kink waves as obtained by previous studies (Dymova \& Ruderman 2005, Díaz et al. 2010) if the external density inhomogeneity is similar to or smaller than the internal one. Stronger density inhomogeneity in the external medium significantly alters the frequency ratio for wider tubes, therefore the thin flux tube approximation can be used with caution in this case. However, external plasma is usually hotter than the plasma inside prominence threads, which means that the external density inhomogeneity should be smaller than the internal one. Then it justifies the applicability of the thin flux tube approximation in prominence seismology. 


\section{Discussion and conclusion}

The period ratio of second and first harmonics is near 2 in weakly inhomogeneous coronal loops; however, it is quite different in the case of prominences. Considering piecewise profiles of density concentration at the tube midpoint, several authors have found that the ratio tends to 3. First, Joarder \& Roberts (1992b) found that the asymptotic frequency of internal kink mode is proportional to $2 n+1$ (see Eq. (21) in their paper), which yields that the period ratio of first and second harmonics of kink mode is 3 in the prominence case. Second, one can see in Figs. 4 and 5 in Dymova \& Ruderman (2005) that the period ratio tends to 3 when $W / L$ approaches 0.2 , where $W$ is the half length of thread and $L$ the half length of the tube itself. Third, Fig. 6 in Díaz et al. (2010) shows that the period ratio of first and second harmonics of kink mode tends to 3 for $W / L>0.1$. Joarder \& Roberts (1992b), Dymova \& Ruderman (2005), and Díaz et al. (2010) did not explicitly note this fact and consequently did not explain why the period ratio tends towards 3 in prominences.

In this paper, we showed that the tendency of period ratio towards 3 is the result of an analogy between prominence oscillations and oscillations of quantum harmonic oscillator. The density enhancement at the midpoint of the longer tube, which appears as a prominence thread, plays a role as the potential energy. We used the method of separation of variables and solved the Sturm-Liouville problem of bounded oscillations in a prominence thread with a longitudinally inhomogeneous density of parabolic profile. We found that the spatial variation of total pressure along the tube axis is governed by the stationary Schrödinger equation, where the term with the longitudinal inhomogeneity of density stands for the potential energy. The equation is transformed into the equation of parabolic cylinder for the parabolic profile of the density. Consequently, the solutions are found in terms of Hermite polynomials, which are a set of orthogonal polynomials over the domain $(-\infty, \infty)$. Therefore, the solutions form an orthogonal basis of the Hilbert space.

The solutions are bounded in infinity, therefore the oscillations are trapped inside the thread and hence are localized much higher than the footpoints. Then, using the continuity of velocity and total pressure at the tube surface, we derived the transcendental dispersion equation for MHD oscillations in terms of Bessel functions. The dispersion equation was solved analytically in a thin flux tube approximation for kink waves. We found that the normalized frequency of a fundamental kink mode depends on the inhomogeneity parameter $\alpha$ as $\omega_{n} \sim \alpha(2 n+1)$, where $n$ is the longitudinal wave mode. This expression shows that the stronger inhomogeneity leads to the higher frequency of kink waves. However, the ratio of second and first harmonics of kink waves do not significantly depend on $\alpha$ in the thin tube approximation, and it tends to 3 as suggested by the analogy with quantum mechanics.

We solved the dispersion equation numerically for kink and sausage waves. The numerical solutions agree with the analytical estimations. We found that the frequency ratio of second and first harmonics of kink waves tends to 3 in thin flux tubes. The ratio depends slightly on the width to length ratio of the tube (Fig. 4). The wider tubes lead to a somewhat lower ratio of the frequencies when the inhomogeneity parameter inside and outside the tube is the same. Therefore, the thin flux tube approximation is a good approximation for the estimation of the ratio in this case. However, when the external inhomogeneity of plasma density is stronger, the ratio significantly depends on the inhomogeneity for wider tubes and the thin tube approximation may fail. Numerical solution of the dispersion equation shows that the frequency ratio slightly depends on the longitudinal inhomogeneity and decreased for stronger $\alpha$ (Fig. 5) for thin tubes, which is in good agreement with analytical formulas.

The calculation in this paper was performed for straight tubes, while the curvature may significantly affect the oscillation spectrum (Selwa et al. 2005; Díaz et al. 2006). It would be also interesting to study the case of parabolic longitudinal inhomogeneity, when the denser plasma is located near the tube ends. In this case, the tube is the analogue of coronal loops rather than of prominence threads. Both problems should be studied in the future.

Acknowledgements. This work was supported by the Austrian Fonds zur Förderung der Wissenschaftlichen Forschung (project P26181-N27), by the European FP7-PEOPLE-2010-IRSES-269299 project- SOLSPANET and by Shota Rustaveli National Science Foundation grant DO/59/6-310/13. The work of TZ was also supported by Shota Rustaveli National Science Foundation grant DI/14/6-310/12. The work of IZh was supported by the Bulgarian Science Fund under project CSTC/INDIA 01/7.

\section{References}

Abramowitz, M., \& Stegun, I. A. 1964, Handbook of Mathematical Functions (Washington: National Bureau of Standards)

Andries, J., Goossens, M., Hollweg, J. V., \& Arregui, I. 2005a, A\&A, 430, 1109 Andries, J., Arregui, I., \& Goossens, M. 2005b, ApJ, 624, L57

Andries, J., van Doorsselaere, T., Roberts, B., et al. 2009, Space Sci. Rev., 149, 3

Arregui, I., Soler, R., Ballester, J. L., \& Wright, A. N. 2011, A\&A, 533, A60

Arregui, I., Oliver, R., \& Ballester, J. L. 2012, Liv. Rev. Sol. Phys., 9, 2

De Moortel, I., \& Brady, C. S. 2007, A\&A, 664, 1210

Díaz, A. J., Oliver, R., \& Ballester, J. L. 2002, ApJ, 580, 550

Díaz, A. J., Oliver, R., Ballester, J. L., \& Roberts, B. 2004, A\&A, 424, 1055

Díaz, A. J., Oliver, R., \& Ballester, J. L. 2005, A\&A, 440, 1167

Diáz, A. J., Zaqarashvili, T. V., \& Roberts, B. 2006, A\&A, 455, 709

Díaz, A. J., Oliver, R., \& Ballester, J. L. 2010, ApJ, 725, 1742

Dymova, M. V., \& Ruderman, M. S. 2005, Sol. Phys., 229, 79

Edwin, P. M., \& Roberts, B. 1983, Sol. Phys., 88, 179

Griffiths, D. J. 2004, Introduction to Quantum Mechanics, 2nd edn. (Upper Saddle River, NJ 07458: Prentice Hall)

Inglis, A. R., \& Nakariakov, V. M. 2009, A\&A, 493, 259

Joarder, P. S., \& Roberts, B. 1992a, A\&A, 256, 264

Joarder, P. S., \& Roberts, B. 1992b, A\&A, 261, 625

Joarder, P. S., Nakariakov, V. M., \& Roberts, B. 1997, Sol. Phys., 176, 285

Labrosse, N., Heinzel, P., Vial, J.-C., et al. 2010, Space Sci. Rev., 151, 243

Lin, Y., Engvold, O., Rouppe van der Voort, L. H. M., Wiik, J. E., \& Berger, T. E. 2005, Sol. Phys., 226, 239

Lin, Y., Engvold, O., Rouppe van der Voort, L. H. M., \& van Noort, M. 2007, Sol. Phys., 246, 65

Lin, Y., Soler, R., Engvold, O., et al. 2009, ApJ, 704, 870

Mackay, D. H., Karpen, J. T., Ballester, J. L., Schmieder, B., \& Aulanier, G. 2010, Space Sci. Rev., 151, 333.

McEwan, M. P., Donnelly, G. R., Díaz, A. J., \& Roberts, B. 2006, A\&A, 460, 893

Nakariakov, V. M., \& Oraevsky, V. N. 1995, Sol. Phys., 160, 289

Nakariakov, V. M., Hornsey, C., \& Melnikov, V. F. 2012, ApJ, 761, 134

Oliver, R. 2009, Space Sci. Rev., 149, 175

Oliver, R., \& Ballester, J. L. 2002, Sol. Phys., 206, 45

Oliver, R., Ballester, J. L., Hood, A. W., \& Priest, E. R. 1993, ApJ, 409, 809

Roberts, B. 1991, Geophysical and Astrophysical Fluid Dynamics, 62, 83

Selwa, M., Murawski, K., Solanki, S. K., Wang, T. J., \& Tóth, G. 2005, A\&A, 440,385

Srivastava, A. K., Zaqarashvili, T. V., Uddin, W., Dwivedi, B. N., \& Kumar, P. 2008, MNRAS, 388, 1899

Srivastava, A. K., Dwivedi, B. N., \& Kumar, M. 2013, Ap\&SS, 345, 25

Terradas, J., Arregui, I., Oliver, R., \& Ballester, J. L. 2008, ApJ, 678, L153

Van Doorsselaere T., Debosscher A., Andries J., \& Poedts S. 2004, A\&A, 424, 1065

Van Doorsselaere, T., Nakariakov, V. M., \& Verwichte, E. 2007, A\&A, 473, 959

Verwichte, E., Nakariakov, V. M., Ofman, L., \& DeLuca, E. E. 2004, Sol. Phys., 223, 77

Zaqarashvili, T. V., \& Murawski, K. 2007, A\&A, 470, 353

Zaqarashvili, T. V., Melnik, V. N., Brazhenko, A. I., et al. 2013, A\&A, 555, A55 